\title{
Design of an Interactive Game for Teaching War Ethics
}

\author{
Nikolaos Doukas ${ }^{1,2}$, Athanasios Drigas ${ }^{3}$, and Nikolaos G. Bardis ${ }^{1,3,4}$ \\ ${ }^{1}$ Hellenic Army Academy, Department of Mathematics \\ and Engineering Science, Vari - 16673, Greece \\ ${ }^{2}$ Hellenic Air Force Academy, Department of Computer Sciences, \\ Dekelia Air Base, Tatoi, Metamorfosi 144 51, Greece \\ ${ }^{3}$ Institute of Informatics \& Telecommunications, Net Media Lab \\ N.C.S.R. Demokritos, Agia Paraskevi, 153 10, Athens, Greece \\ ${ }^{4}$ Hellenic Naval Academy, Terma Hadjikyriakou Avenue, Piraeus - 18539 \\ doukasn@sse.gr, nikolaos@doukas.net.gr, \\ dr@it.demokritos.gr, bardis@ieee.org
}

\begin{abstract}
This work focuses on the use of computer simulations and computer games for the training of prospective and serving Armed Forces officers. A study of the use of computer simulations and games for teaching and training in the context of both the Armed Forces and civilian applications is presented. The study leads to high level specifications for the design of the proposed training game. The functional design of the proposed game is then explained and its rules are listed. The design is shown to offer the flexibility required for the teaching courses it is meant to serve. Further research necessary for the development of optimization algorithms necessary for the design is described.
\end{abstract}

Keywords: Technology enhanced learning, teaching games, winning strategies, computer simulations, pursuit and evasion, differential games.

\section{Introduction}

The use of computer simulations has been extensively exploited for enhancing the training of Armed Forces officers in decision making for tactical situations [1]. Teaching of humanities courses like Philosophy or War Ethics still has a lot to benefit from the introduction of e-Learning technologies into the everyday didactic routine [2]. Adopting a technology enhanced learning approach for explaining the theoretical principles involved to students and assessing their understanding, is an intriguing problem. Solutions to this problem require purpose designed systems that address the particular characteristics of specific target student groups. This paper is concerned with the application of technology enhanced learning for the teaching of war ethics to prospective officers of the Armed Forces. More particularly, it is concerned with the technical part of the design of an interactive, on-line war game capable of assessing if students are able of maintaining ethical rules of engagement in conflict while maintaining the required high standards of strategic and tactical efficiency. The use of on-paper war games in teaching Armed Forces cadets is a topic of increasing interest [3]. The problems relating to the assessment of electronic War Games have been 
extensively analyzed in [4] and a multidimensional assessment roadmap has been proposed. In the same study, a very simple sample scenario and preliminary design principles for such a game have been listed.

The aim of this paper is to present a high level functional design of an interactive, on-line, multiplayer game that will be capable of supporting the training prospective officers of the armed forces on the war ethics and maintaining international law governing the rules of armed conflict. The game incorporates tactical and strategic aspects so as to enable students to appreciate the difficulties of abiding by ethical rules while achieving their military goals and devise methods for overcoming such difficulties. The design of the game allows users to compose new crisis scenarios, to determine the rules that are applicable in each case, to assign the importance of the different aspects of the game to the final scoring of the participants and of course to play the game. Algorithms developed in the context of the game can also be used as driving forces for decision support tools.

In Section 2, existing work on the field of games design for teaching and training purposes for both military and civilian contexts is presented. In Section 3, the functional specifications for the present work are explained and the high level functional design for the proposed war game is given. In Section 4, the advantages of the proposed design are outlined. It is hence demonstrated that this design offers significant flexibility for instructors and effectively supports the teaching of the required topics of the curriculum. Finally, a roadmap for the implementation of the game and its assessment in real teaching is given.

\section{Interactive Games for Training in Decision Making}

The use of games for teaching specialized skills to prospective or serving officers of the armed forces is a well accepted approach ([1], [2], [3], [4], [7], [8], [9]). Officers, as well as many other professionals, need to be able of taking accurate decisions within very strict time limits while taking into account a large amount of possibly uncertain information. This is a very complex task and it is hence difficult to train the personnel simply on a theoretical basis. Such decisions involve subjective evaluation criteria, constraints of a physical or legal nature, assessment of imprecise data and selection from multiple action alternatives. For the case of military operations, the situation the officer has to be trained in is called the "Fog of War" [2], a term that illustrates the fact that the commanding officer always has an impaired view of the complete picture of the field of action.

The use of computer simulations for training (or aiding) personnel to efficiently cope with this kind of situations is always impeded by the fact that both the decision space of such problems, as well as the parameter space upon which these decisions are based are neither finite, nor can they be unambiguously defined [1]. What is proposed in literature therefore is the use of mathematical optimization to follow the situation as it evolves in real time or pseudo-real time ([1], [6], [9], [11]).

Depending on the training required, different types of mathematical modeling may be employed. Pursuit and Evasion situations may be considered as an example of a Differential Game [6]. Pursuit and Evasion games include the "Guarding the target 
problem, deadline games and patrolling games [6]. Their general aim is to guard a target, which is an area on the plane, from the attacks launched by the evader. Such problems may be formulated in both continuous and discrete versions. A discrete guarding game played by two players based on a directed or undirected graph is an NP hard problem [6]. When considered under restrictions, such as a limited size plane, encompassing an undirected graph, analytical answers as to the optimal strategy that a player should follow may be derived [7]. In its simpler form, a pursuit game is a zero-sum game, in that the goals of the players are conflicting (player A wants to occupy space $S$, currently occupied by B etc). A less simplistic approach to the design of pursuit games is the non-zero sum case where the goals of the players may not always be conflicting [12].

For a three player discrete game where coalitions are possible, probabilistic analysis, rather than the adoption of an optimal analysis is proposed [8]. Allowing explicit coalitions in games completely alters the theoretical perspective of the game, since the actions of the allied players are independent and may encompass different field information. Defeating a coalition may involve different strategies, such as defeating each ally at different times [8].

A further distinction needs to be made between full information and partial information games. In full information games, all players have full knowledge of all the opponent's forces, as well as all their past moves. In partial information games, players lack knowledge of their opponent's past moves and their opponent's present situation. A player must hence hypothesize about all these parameters and the complexity of the analysis required to derive an optimal solution increases dramatically [9]. Partial information games are better suited to model the fog of war concept.

Continuous modeling of pursuit situations is also possible. In this case pursuers and targets are moving in continuous space and time and the requirements are minimizations of the distances and times subject to the physical constraints of the hardware being used [10]. The scope of the game in this case becomes an optimization of the speed of reaction of the players and deviates from the aim of this work which is targeted to the philosophical aspects of applying the ethics of war rules, subject to the strategic and tactical aims, as well as to the limitations of the fog of war.

Games are an intriguing tool for training personnel on responding to hard to solve problems in real time and this may be verified by the amount of research devoted to this topic [11]. In this section a selection of issues concerning the design of games was presented. This leads to a clearer view on how the design of a game suitable for the teaching of ethics should be approached.

\section{Design of the Game}

The aim of this work was to design a game that is suitable for teaching ethical rules. Given that this game was targeted for application in the case of prospective Armed Forces officers, it needed to be able to keep the students - players constantly aware of three additional very important aspects of their mission, namely its strategic and its tactical goals as well as the inevitable impairment of the fog of war. It is proposed to be a partial information game of more than two players with the possibility of coalitions. 


\subsection{The Concept of the Game}

More specifically the proposed game is based on a grid, similar in concept with a naval battle game [4] or chess. The main difference from these games is that the proposed war game uses a larger grid with significantly finer resolution. The players of the game belong to one of two groups:

- Tactical army: These are players that represent the armed forces of a state and are hence bound by laws, ethical rules etc.

- Terrorist groups: These players represent forces that are not bound by ethics or rules and their behavior is totally unpredictable. Such players may be human participants or may be automatically handled by the computer program.

In addition to the above, there are two additional categories of players that may be active on the board or be implicitly represented:

- Strategic command: Represent the leaders of tactical armies and set military targets and assessment parameters

- Civilian population: Represent non-fighting individuals that exist on parts of the grid. Despite the fact that they are not active militarily, they may influence the outcome of a battle in various ways, e.g by storing weapons for one player or preventing a player from attacking a specific target.

The two last player categories are typically game parameters set by the instructor (implicit participation) or are active characters that are managed by either the instructor or the computer. Two or more players may belong to an alliance.

Each player possesses a number of pawns that are placed on specific positions on the grid. The grid is a discrete space, i.e. pawns may reside only on the vertices of the grid. Areas on the grid represent different geographical areas (ground, sea, buildings, difficult to access areas). Each pawn represents a specific type of forces (infantry, armored vehicles, ships, planes etc). The pawns may be laid on the grid by either the instructor or the player at the beginning of the game. Civilian pawns are also positioned on the grid by the instructor.

Each player plays on their own computer screen. On their own screen they are capable of seeing their own pawns and the pawns of their allies. Each of the pawns of a player also conveys intelligence information to their headquarters. In practice, this means that a player can also observe on their screen information about their opponents' pawns that their own pawns can see. An infantry unit will hence give its owner visibility over a small area around it, while an airplane will give visibility over a larger area.

\subsection{Playing the Game}

Each player plays in turn and moves a number of pawns or uses a number of weapons. Each pawn may move a certain distance on the grid according to its type. The number of pawns moved at each turn by each player is a parameter set by the instructor. Each pawn carries a limited number of weapons, depending on its type. Each weapon is effective against specific types of adversary pawns. A player may choose to either destroy or arrest pawns that are in range of their weapons. The use of certain types of 
weapons may be prohibited or impossible in certain areas. Weapons may be stored in certain positions on the grid. Such weapons are accessible to anybody occupying that part of the grid.

\subsection{Pawn Movement Limitations}

A player is given an initial amount of credit at the beginning of the game. Each pawn move has a cost that is deducted from their credit. Similarly, each weapon use also has a cost. A player may not move a pawn or use a weapon if they do not have enough credit left for this move. Certain positions on the grid are not allowed for certain types of pawns (e.g. the sea for infantry).

\subsection{Definition of the Mission}

Players are assigned missions that they need to achieve. A mission may involve occupying an area on the grid, arresting a number of adversary pawns, destroying a number of adversary pawns etc. Pawns that have been arrested and are prisoners of a player, incur a steady cost upon that player.

\subsection{Scores and Eviction of Players from the Game}

Each part of a mission that is achieved carries a certain number of points towards the final assessment of the player. A player also adds points to their score according to the credit they have left at the end of the game. A player loses points by not having achieved certain parts of their mission at the end of the game, by destroying civilians or allies and by using certain weapons in certain areas. These weapon - area limitations are instructor set parameters.

A player is evicted from the game if certain weapons in certain areas, if they use a weapon on an area with no targets or if they disobey any other instructor set rules. The player is also evicted from the game if a certain percentage of their pawn is destroyed or captured. The game finishes when a player achieves a certain percentage of the goals of their mission or if there is only one player left in the game.

For the case when some players assume the role of terrorists, scoring becomes asymmetrical, i.e. some players may score points for the same actions that cost points to other players.

\section{Assessment of the Design and Future Work}

The design of the game that was presented in a previous section represents a realistic model for the situations in which the players will have to apply the ethics of war principles that they have been taught. The ethical aspect of the game has been expressed as rules that either cost a player points or cause the "sudden death" of a player, i.e. their immediate expulsion from the game. This way, ethical rules could be measured on the same scale as other objectives of the game such as the completion of the player's mission or the credit points that the player has had to spend in order to pursue their mission. This model represents a real world situation where deviation from the ethical rules incurs costs upon real world players in the form of social pressure, social exclusion as well as legal proceedings. 
The proposed design gives extensive liberty to the instructor to implement the game so as to match the priorities of their own classroom teaching, the particular aspects of the theory that they wish to stress or the strengths and weaknesses of a particular group of students. The instructor also has full liberty to participate in the game by assuming unconventional roles, such as that of a terrorist group, groups of civilians or the strategic leader of the players. The design also allows the game to change its scope, from a training exercise to a decision support platform. This is feasible by the use of suitable optimization algorithms that derive feasible, optimal or suboptimal sequences of movements for completing missions, based on mathematical analysis or heuristic rules.

There are still several theoretical issues regarding this work that need to be studied. These have to do with algorithms for producing strategic and tactical paths for dealing with specific situations with which the players may be faced. Algorithms will have to be designed based on both analytical and heuristic approaches. It has been shown in the literature surveyed that optimal solutions will not in general be feasible. The feasibility or infeasibility of seeking an optimal solution will have to be studied. Additionally, the calculation of limits in performance may be sought that can be used to compare the relative merits of heuristic solutions. The calculation of lower bounds that permit the determination of realistic tactical and strategic goals are also of interest to this work. Such a lower bound is derived in [8] for the case of a Cop and Robber game, where it is shown that depending on the distance between the two players, the game may be futile for either the Cop or the Robber.

The final and maybe most important step of this study will be the use of the game in the classroom and the assessment of its impact towards facilitating the teaching and improving the students' understanding of the topic. Additional interest is focused on the effectiveness of the strategies that the optimization algorithms will produce for the players that are automatically controlled (e.g. the terrorist groups).

\section{Conclusions}

A study on the use of computer games in teaching prospective Armed Forces officers. Existing work relating to the design of war games and training games were presented. The high level design of a training game was presented that is suitable for use in the teaching of war ethics to students. The game was designed to be able to assess the player skills in maintaining ethical rules and international laws on armed conflict while still achieving the strategic and tactical aims of their missions. The broad rules of the game were presented. The design was shown to offer flexibility to the instructors, allowing them to adapt the game to the requirements of their own teaching. Directions for further development of the game were presented, in both theoretical and applied aspects.

\section{References}

1. Hicks, J., Petrov, V., Stoyen, A., Zhu, Q.: Intelligent agent-based software architecture for combat performance under overwhelming information inflow and uncertainty. In: Seventh IEEE International Conference on Engineering of Complex Computer Systems, pp. 200 $210(2001)$ 
2. Lekea, I.: New Technologies, Distance Learning, Internet and Games; their contribution in a New Approach to the Educational practice. The Case Study of the Philosophy Course in the Hellenic Army Academy. In: 1st Hellenic Conference of Educational Sciences, Athens (2009)

3. Lekea, I., Sotiriadis, P.: Designing an Educational Game for Aircraft Engineers; Theoretical Experience and Effectiveness in Teaching Practice. In: 1st Hellenic Conference of Educational Sciences, Athens (2009)

4. Andreatos, A., Lekea, I.: Technological Evolution and Pedagogical Reapproach to Effective Learning Using Games within the Hellenic Air Force Academy. The Case Study of the Philosophy Course in the Hellenic Army Academy. In: Readings in Technology and Education: Proceedings of ICICTE, pp. 799-811 (2009)

5. Naval battle on-line game, http: / / www . energynet . net/games / battle/

6. Fomin, F., Golovach, P., et al.: How to Guard a Graph? Technical Report Nr. 605, Department of Computer Science, ETH Zurich (2008)

7. Alonso, L., Reingold, E.: Lower bounds for Cops and Robbers Pursuit (2008), http://emr.cs.uiuc.edu/ reingold/copsandrobber.pdf

8. Dshalalow, J., Treerattrakoon, A.: Set-Theoretic Inequalities in Stochastic Noncooperative Games with Coalition. Journal of Inequalities and Applications, Article ID 713642 (2008)

9. Hespanh, J., Ateskan, Y., Kizilocak, H.: Deception in Non-Cooperative Games with Partial Information. In: Proc. of the 2nd DARPA-JFACC Symposium on Advances in Enterprise Control, pp. 1-9 (2000)

10. Looker, J.: Minimum Paths to Interception of a Moving Target when Constrained by Turning Radius. Publication DSTO-TR-2227, Defence Science and Technology Organization, Air Operations Division, Australian Dept. of Defense (2008)

11. Fraenkel, A.: Combinatorial Games: Selected Bibliography with a Succinct Gourmet Introduction. The Electronic Journal of Combinatorics (2009)

12. Kwon, O., Tarashnina, S.: On a Time-Consistent Solution of a Cooperative Differential Time-Optimal Pursuit Game. J. Korean Math. Soc. 39(5), 745-764 (2002) 